\title{
Polygenic Hyperlipidemias and Coronary Artery Disease Risk
}

\section{FinnGen Project}

2020-04

FinnGen Project , Ripatti , P , Rämö , J T , Mars , N J , Fu , Y , Lin , J , Söderlund , S , Benner , C , Surakka , I , Kiiskinen, T , Havulinna, A S , Palta , P , Freimer , N B , Widén , E , Salomaa , V , Tukiainen , T , Pirinen , M , Palotie , A , Taskinen , M-R \& Ripatti , S 2020 , ' Polygenic Hyperlipidemias and Coronary Artery Disease Risk ' , Circulation-Genomic and precision medicine , vol. 13 , no. 2 , pp. 59-65 . https://doi.org/10.1161/CIRCGEN.119.002725

http://hdl.handle.net/10138/314469

https://doi.org/10.1161/CIRCGEN.119.002725

cc_by

publishedVersion

Downloaded from Helda, University of Helsinki institutional repository.

This is an electronic reprint of the original article.

This reprint may differ from the original in pagination and typographic detail.

Please cite the original version. 


\title{
Polygenic Hyperlipidemias and Coronary Artery Disease Risk
}

\begin{abstract}
Pietari Ripatti®, MD; Joel T. Rämö®D, MD; Nina J. Mars, MD, PhD; Yu Fu, MSc; Jake Lin, PhD; Sanni Söderlund, MD, PhD; Christian Benner, PhD; Ida Surakka, PhD; Tuomo Kiiskinen, MD; Aki S. Havulinna, PhD; Priit Palta, PhD;

Nelson B. Freimer, MD, PhD; Elisabeth Widén, MD, PhD; Veikko Salomaa, MD, PhD; Taru Tukiainen, PhD; Matti Pirinen, PhD; Aarno Palotie, MD, PhD; Marja-Riitta Taskinen®, MD, PhD*; Samuli Ripatti®, PhD*; on behalf of FinnGen†
\end{abstract}

BACKGROUND: Hyperlipidemia is a highly heritable risk factor for coronary artery disease (CAD). While monogenic familial hypercholesterolemia associates with severely increased CAD risk, it remains less clear to what extent a high polygenic load of a large number of LDL (low-density lipoprotein) cholesterol (LDL-C) or triglyceride (TG)-increasing variants associates with increased CAD risk.

METHODS: We derived polygenic risk scores (PRSs) with $\approx 6 \mathrm{M}$ variants separately for LDL-C and TG with weights from a UK Biobank-based genome-wide association study with $\approx 324 \mathrm{~K}$ samples. We evaluated the impact of polygenic hypercholesterolemia and hypertriglyceridemia to lipid levels in 27039 individuals from the National FINRISK Study (FINRISK) cohort and to CAD risk in 135638 individuals (13 753 CAD cases) from the FinnGen project (FinnGen).

RESULTS: In FINRISK, median LDL-C was $3.39(95 \% \mathrm{Cl}$, 3.38-3.40) mmol/L, and it ranged from $2.87(95 \% \mathrm{Cl}$, 2.82-2.94) to $3.78(95 \% \mathrm{Cl}, 3.71-3.83) \mathrm{mmol} / \mathrm{L}$ between the lowest and highest $5 \%$ of the LDL-C PRS distribution. Median TG was 1.19 $(95 \% \mathrm{Cl}, 1.18-1.20) \mathrm{mmol} / \mathrm{L}$, ranging from $0.97(95 \% \mathrm{Cl}, 0.94-1.00)$ to $1.55(95 \% \mathrm{Cl}, 1.48-1.61) \mathrm{mmol} / \mathrm{L}$ with the TG PRS. In FinnGen, comparing the highest 5\% of the PRS to the lowest 95\%, CAD odds ratio was $1.36(95 \% \mathrm{Cl}, 1.24-1.49)$ for the LDL-C PRS and $1.31(95 \% \mathrm{Cl}, 1.19-1.43)$ for the TG PRS. These estimates were only slightly attenuated when adjusting for a CAD PRS (odds ratio, 1.26 [95\% Cl, 1.16-1.38] for LDL-C and 1.24 [95\% Cl, 1.13-1.36] for TG PRS).

CONCLUSIONS: The CAD risk associated with a high polygenic load for lipid-increasing variants was proportional to their impact on lipid levels and partially overlapping with a CAD PRS. In contrast with a PRS for CAD, the lipid PRSs point to known and directly modifiable risk factors providing additional guidance for clinical translation.

Key Words: coronary artery disease $\mathbf{m}$ humans $\boldsymbol{\|}$ hypercholesterolemia $\boldsymbol{\|}$ hypertriglyceridemia $\boldsymbol{\|}$ risk factors

H ypercholesterolemia, particularly high LDL (lowdensity lipoprotein) cholesterol (LDL-C), is an established, heritable, and treatable risk factor for coronary artery disease (CAD)., ${ }^{1,2}$ Additionally, accumulating evidence suggests that increased triglycerides (TGs; hypertriglyceridemia) are causally linked to $C A D{ }^{3-5}$

Increased levels of both LDL-C and TGs result from a combination of genetic and nongenetic factors. ${ }^{6,7}$
Genetic factors include rare highly penetrant variants and a long tail of common variants with smaller effect sizes. While pathogenic variants in the LDLR, PCSK9, and $A P O B$ genes cause familial hypercholesterolemia, it has also been suggested that similarly high LDL-C levels could result from a high polygenic burden of LDLC-increasing variants., ${ }^{8,9}$ Monogenic familial hypercholesterolemia with an identified mutation associates with

Correspondence to: Samuli Ripatti, PhD, University of Helsinki, Tukholmankatu 8, Fl-00290 Helsinki, Finland. Email samuli.ripatti@helsinki.fi

*Drs Taskinen and Ripatti contributed equally to this work as senior authors.

tA list of all FinnGen study participants is given in the Data Supplement.

The Data Supplement is available at https://www.ahajournals.org/doi/suppl/10.1161/CIRCGEN.119.002725.

For Sources of Funding and Disclosures, see page 65.

(C) 2020 The Authors. Circulation: Genomic and Precision Medicine is published on behalf of the American Heart Association, Inc., by Wolters Kluwer Health, Inc. This is an open access article under the terms of the Creative Commons Attribution Non-Commercial-NoDerivs License, which permits use, distribution, and reproduction in any medium, provided that the original work is properly cited, the use is noncommercial, and no modifications or adaptations are made.

Circulation: Genomic and Precision Medicine is available at www.ahajournals.org/journal/circgen 


\section{Nonstandard Abbreviations and Acronyms}

$\begin{array}{ll}\text { CAD } & \text { coronary artery disease } \\ \text { HDL } & \text { high-density lipoprotein } \\ \text { LDL } & \text { low-density lipoprotein } \\ \text { LDL-C } & \text { low-density lipoprotein cholesterol } \\ \text { OR } & \text { odds ratio } \\ \text { PRS } & \text { polygenic risk score } \\ \text { TG } & \text { triglyceride }\end{array}$

a higher $C A D$ risk than expected on the basis of a single LDL-C measurement. ${ }^{10}$ While previous studies have linked a handful of common variants to both increased LDL-C and CAD risk, the contribution of an accumulation of a large number of LDL-C-increasing alleles to CAD risk has remained unknown. ${ }^{11}$

Similarly to hypercholesterolemia, both polygenic burden and highly penetrant variants contribute to hypertriglyceridemia. ${ }^{6}$ However, highly penetrant variants underlying hypertriglyceridemia are much fewer and rare (estimated population prevalence, 1:1 000000 ). ${ }^{6}$ On the contrary, many individuals with hypertriglyceridemia have a high polygenic burden of TG-increasing variants. ${ }^{6}$ Unlike LDL-C, it is unknown whether genetically increased TGs confer higher CAD risk than nongenetic hypertriglyceridemia. Genetics supporting a causal link between hypertriglyceridemia and CAD, and the evidence for beneficial therapeutic reducing of TGs to reduce cardiovascular disease risk, however, highlight the potential also for association between polygenic load of TG elevating alleles and CAD risk. ${ }^{3-5,12,13}$
In this cohort study of 27039 individuals from the Finnish National FINRISK Study (FINRISK) population cohort with lipid measurements, and 135638 individuals including 13753 CAD cases from the FinnGen project (FinnGen), we evaluated the impact of high polygenic $L D L-C$ and $T G$ to $\mathrm{CAD}$ risk. We developed separate genome-wide polygenic risk scores (PRSs) for both LDL-C and TG to define polygenic hypercholesterolemia and hypertriglyceridemia. First, we tested to what extent PRSs for LDL-C and TG associate with measured lipid levels. Second, we tested to what degree polygenic hypercholesterolemia and polygenic hypertriglyceridemia associate with increased risk for CAD.

\section{METHODS}

Because of the sensitive nature of the data collected for this study, requests to access the data set from qualified researchers trained in human subject confidentiality protocols may be submitted through the Finnish Biobanks' FinnBB portal (https://finbb.fi/) for FinnGen at https://www.ukbiobank.ac.uk/researchers/ for the UK Biobank (UKBB) and at https://www.thl.fi/biobank/researchers for the GeneRISK study (GeneRISK) and FINRISK.

The Coordinating Ethics Committee of the Helsinki and Uusimaa Hospital District approved the FinnGen project (No. HUS/990/2017), the GeneRISK study, and the 2007 and 2012 FINRISK surveys. Earlier FINRISK surveys were approved by various ethics committees. ${ }^{14}$ The North West Multi-Centre Research Ethics Committee approved the UKBB study. Written informed consent was obtained from all participants except the 1992 FINRISK survey, for which verbal informed consent was obtained as required by legislation and ethics committees at the time.

The full Methods are available as the Data Supplement.

Table 1. Clinical and Metabolic Characteristics of Individuals

\begin{tabular}{|c|c|c|c|c|}
\hline \multirow[b]{2}{*}{ Characteristics } & \multicolumn{2}{|c|}{ FINRISK } & \multicolumn{2}{|l|}{ FinnGen } \\
\hline & $\mathrm{n}$ & Mean \pm SD & $\mathrm{n}$ & Mean \pm SD \\
\hline $\mathrm{n}$ (men/women) & $27039(12884 / 14155)$ & & $135638(59252 / 76386)$ & \\
\hline CAD, n (\%) & $2750(10.2 \%)$ & & $13753(10.1 \%)$ & \\
\hline Lipid-lowering medication usage, $\mathrm{n}(\%)$ & $1658(6.1 \%)$ & & $42193(31.1 \%)$ & \\
\hline Smoking, $\mathrm{n}(\%)$ & $6739(25 \%)$ & & $19690(22.1 \%)$ & \\
\hline Age, $^{*} y$ & 27039 & $48.9 \pm 13.5$ & 135638 & $60.6 \pm 16.5$ \\
\hline $\mathrm{BMI}, \mathrm{kg} / \mathrm{m}^{2}$ & 26941 & $26.8 \pm 4.69$ & 95528 & $27.2 \pm 5.6$ \\
\hline Total cholesterol, $\mathrm{mmol} / \mathrm{L}$ & 27024 & $5.49 \pm 1.08$ & & \\
\hline LDL-C, mmol/L & 26568 & $3.47 \pm 1.01$ & & \\
\hline Triglyceride, $\mathrm{mmol} / \mathrm{L}$ & 27024 & $1.47 \pm 1.00$ & & \\
\hline $\mathrm{HDL}-\mathrm{C}, \mathrm{mmol} / \mathrm{L}$ & 27024 & $1.44 \pm 0.381$ & & \\
\hline Apolipoprotein B, g/L & 22464 & $0.965 \pm 0.248$ & & \\
\hline Non-HDL-C, mmol/L & 27024 & $4.05 \pm 1.10$ & & \\
\hline Remnant-C, mmol/L & 26568 & $0.630 \pm 0.340$ & & \\
\hline
\end{tabular}

LDL-C was calculated using the Friedewald formula; the effect of lipid-lowering therapy in those using medication at the time of lipid measurement was adjusted for by dividing LDL-C by 0.7 as utilized previously. ${ }^{10}$ FinnGen lacks lipid measurements and lipid-lowering medication usage information. BMI indicates body mass index; CAD, coronary artery disease; FinnGen, The FinnGen project; FINRISK, The National FINRISK Study; HDL-C, highdensity lipoprotein cholesterol; LDL-C, low-density lipoprotein cholesterol; and Remnant-C, remnant cholesterol.

*Age at recruitment for FINRISK and age at end of follow-up for FinnGen. 


\section{RESULTS}

\section{Polygenic Hyperlipidemias and Lipid Levels}

We tested the association between PRSs for LDL-C and TG derived from a genome-wide association study of lipid measurements in the UKBB and lipid levels in the Finnish FINRISK study. FINRISK comprises 27039 individuals randomly drawn from the Finnish population (Table 1). Median LDL-C was 3.39 (95\% Cl, 3.38-3.40) $\mathrm{mmol} / \mathrm{L}$ and TG, $1.19(95 \% \mathrm{Cl}, 1.18-1.20) \mathrm{mmol} / \mathrm{L}$ in the whole cohort with slightly lower values in the more recent collections (Figure I in the Data Supplement).

The PRSs consisted of 6 million markers and explained $5.4 \%$ (adjusted $r^{2}$ ) of variation in LDL-C and $5.1 \%$ in TG. In
FINRISK, median LDL-C was $2.87(95 \% \mathrm{Cl}, 2.82-2.94)$ $\mathrm{mmol} / \mathrm{L}$ in the lowest and $3.78(95 \% \mathrm{Cl}, 3.71-3.83)$ $\mathrm{mmol} / \mathrm{L}$ in the highest $5 \%$ of the LDL-C PRS distribution (Figure 1A). Similarly, median TG was $0.97(95 \% \mathrm{Cl}$, 0.94$1.00) \mathrm{mmol} / \mathrm{L}$ in the lowest and $1.55(95 \% \mathrm{Cl}, 1.48-1.61)$ $\mathrm{mmol} / \mathrm{L}$ in the highest $5 \%$ of the TG PRS distribution (Figure 1B). The increases in LDL-C and TG levels were similar in all FINRISK subcollections (Figure II in the Data Supplement). The correlation between the LDL-C PRS and the TG PRS was low $(r=0.15)$. Subsequently, the cross-trait pleiotropy between the LDL-C and TG PRSs was small as the LDL-C PRS explained 0.7\% (adjusted $r^{2}$ ) of TG levels and the TG PRS $0.3 \%$ of LDL-C levels (Figure III in the
A



B

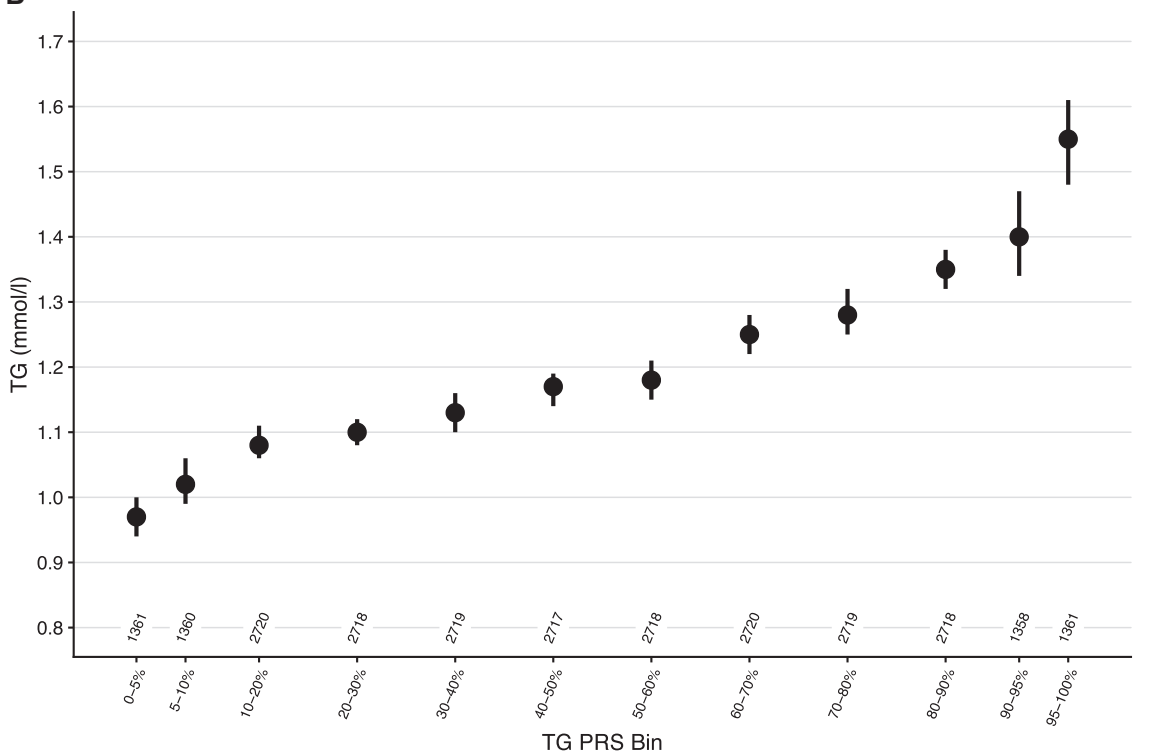

Figure 1. Median lipid levels across the distributions of the lipid polygenic risk scores (PRSs) in the National FINRISK Study (FINRISK) cohort. Numbers of individuals in the PRS bins are reported. Vertical lines represent $95 \%$ Cls. 
Data Supplement). All in all, the LDL-C and TG PRSs had a clear impact on their respective lipid levels.

\section{Polygenic Hyperlipidemias and CAD Risk}

To assess how polygenic hyperlipidemia associates with CAD risk, we analyzed 135638 individuals including 13 753 registry-based CAD cases from the Finnish FinnGen project (Table 1). FinnGen is an aggregation of Finnish prospective epidemiological and disease-based cohorts and hospital biobank samples and, therefore, includes the FINRISK participants. Polygenic hypercholesterolemia associated with increased CAD risk. Compared with the remainder of the population, individuals with the LDL-C PRS in the highest 10\% had 1.4-fold increased CAD risk (odds ratio [OR], $1.41[95 \% \mathrm{Cl}, 1.32-1.50]$ ), and individuals in the highest $5 \%$ also had 1.4 -fold increased risk (OR, 1.36 [95\% Cl, 1.24-1.49]; Figure 2A). CAD prevalence was $52 \%$ higher (12.5\% versus $8.2 \%$ ) between the highest and the lowest $5 \%$ of the LDL-C PRS distribution (Figure 3A). OR for CAD per SD unit increase of the LDL-C PRS was $1.17(95 \% \mathrm{Cl}, 1.15-1.20$; Table 2).

For polygenic hypertriglyceridemia, compared with the remainder of the population, individuals with the TG PRS in the highest 10\% had 1.3-fold increased CAD risk, and individuals in the highest $5 \%$ also 1.3-fold (OR, $1.31[95 \% \mathrm{Cl}, 1.19-1.43])$ increased CAD risk (OR, 1.31 [95\% Cl, 1.22-1.40]; Figure 2B). CAD prevalence was $36 \%$ higher (11.8\% versus $8.7 \%$ ) between the highest and the lowest 5\% of the TG PRS distribution (Figure 3B). OR for CAD per SD unit increase of the TG PRS was $1.12(95 \% \mathrm{Cl}, 1.09-1.14$; Table 2).

We tested whether the lipid PRSs improve CAD risk prediction beyond a similarly derived CAD PRS. Comparing the highest $5 \%$ to the remainder of the population, the effects of the lipid PRSs on CAD risk were attenuated only modestly when adjusted for the CAD PRS (LDL-C PRS OR, 1.26 [95\% Cl, 1.16-1.38] and TG PRS OR, 1.24 [95\% Cl, 1.13-1.36]; Figure 4). The area under the receiver operating characteristic curve of a model with the lipid and CAD PRSs was high (0.879; Table 2). It was, however, similar to a model with only the CAD PRS (0.879; Table 2$)$.

\section{DISCUSSION}

By developing genome-wide PRSs for LDL-C and TG, we evaluated the impact of high genetic risk for these established and causal risk factors of CAD. We showed that high polygenic burden for both LDL-C or TG associated with considerably increased LDL-C and $T G$ levels, respectively. Similarly, polygenic hypercholesterolemia and triglyceridemia associated with significantly increased CAD risk. Furthermore, PRSs for LDL-C and TG were partially overlapping with a PRS for CAD.

Polygenic hypercholesterolemia, in our study, conveyed $0.41 \mathrm{mmol} / \mathrm{L}$ higher LDL-C levels and $36 \%$ higher

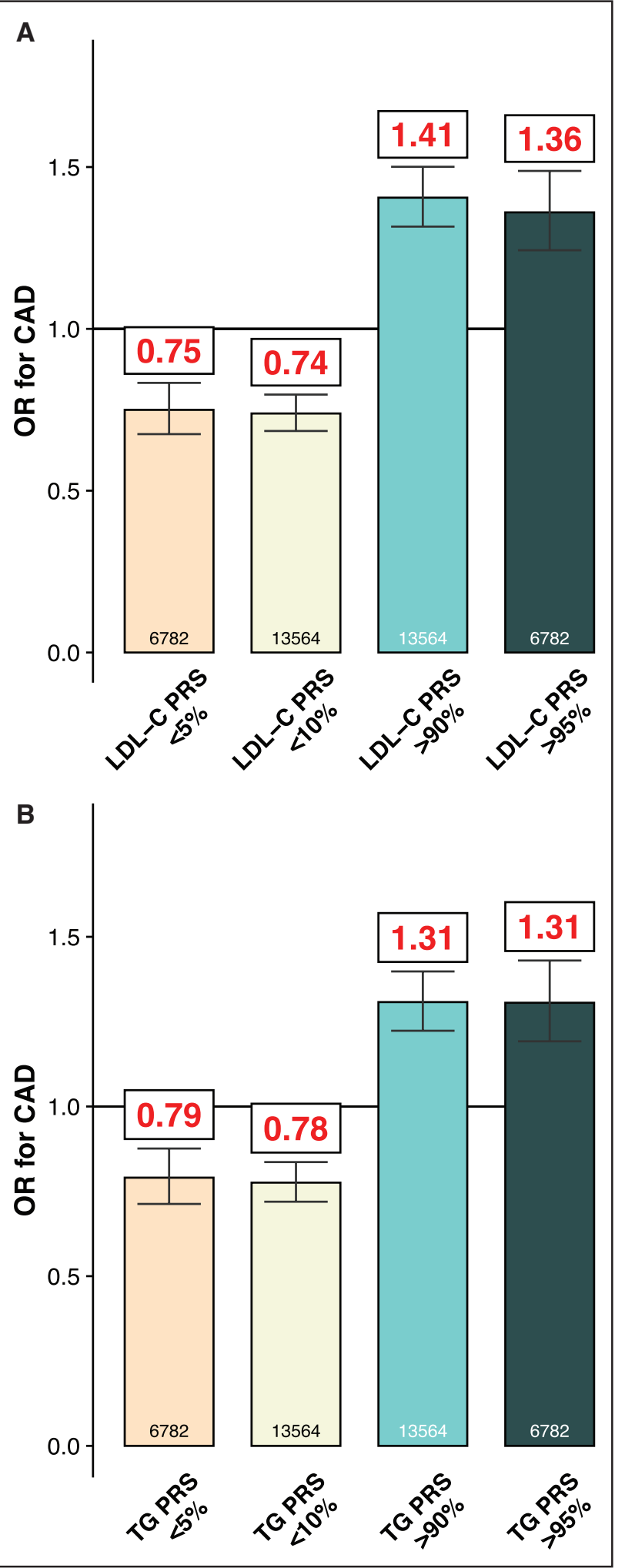

Figure 2. Odds ratios (ORs) for coronary artery disease (CAD) across the lipid polygenic risk score (PRS) distributions in the FinnGen project (FinnGen).

Total numbers of individuals in PRS bins are reported. ORs were estimated using logistic regression adjusted for age, sex, first 10 principal components, and genotyping batch. PRS bins were compared with the remainder of the population. Error bars represent 95\% Cls. 


\section{A}

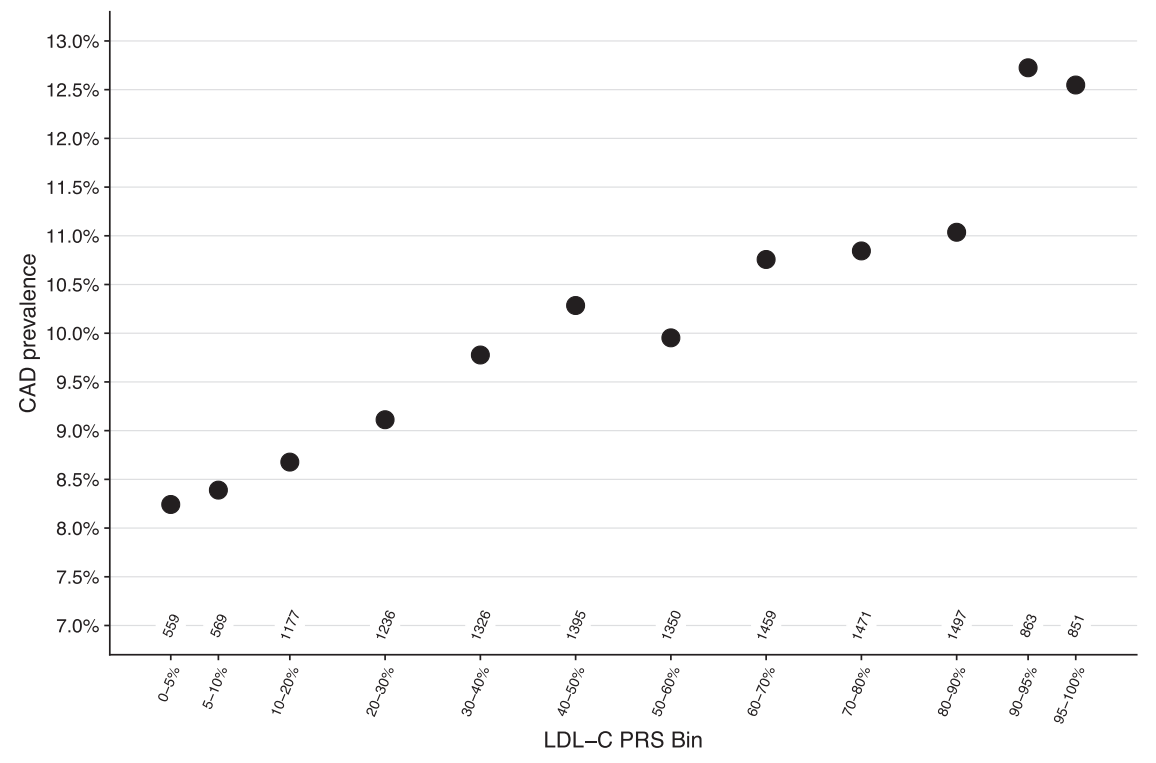

B

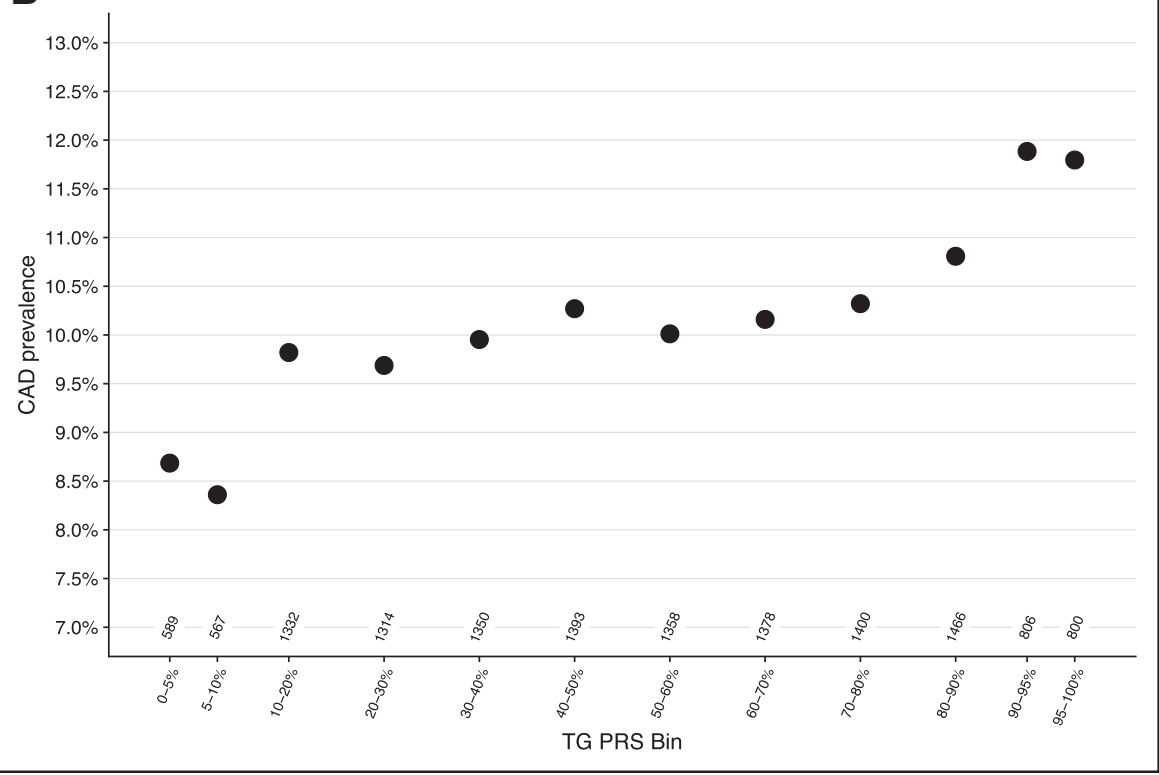

Figure 3. Coronary artery disease (CAD) prevalence across the lipid polygenic risk score (PRS) distributions in the FinnGen project (FinnGen).

Numbers of CAD cases in PRS bins are reported.
CAD risk in the highest $5 \%$ of the LDL-C PRS compared with the remainder of the population. This is considerably lower than the previous estimates of the effects of highimpact $L D L R$ familial hypercholesterolemia mutation on LDL-C levels (2-3 mmol/L) and CAD risk (2.6- to 3.7-fold). ${ }^{10,15,16}$ Going further from the highest $5 \%$ to the highest $1 \%$ of the LDL-C PRS conveyed still only 0.55 $\mathrm{mmol} / \mathrm{L}$ higher LDL-C levels compared with the remainder of the population (Figure IV in the Data Supplement). While the established high-impact $L D L R$ familial hypercholesterolemia mutations directly disrupt LDL receptor function causing lifelong high LDL-C levels, the effect sizes of the individual variants contributing to polygenic hypercholesterolemia are small, and they likely increase $\mathrm{LDL}-\mathrm{C}$ via multiple indirect biological pathways. Whereas monogenic familial hypercholesterolemia is a severe disease with high CAD risk, polygenic hypercholesterolemia, as captured by the current PRSs, seems to have a smaller effect on LDL-C levels and CAD risk. The degree of benefit of lipid-lowering therapies in individuals with polygenic hypercholesterolemia has, therefore, remained largely unknown.

In our study, both LDL-C and TG PRSs associated with CAD risk also when adjusted for a CAD PRS. The key difference between intermediate biomarker PRSs (such as the lipid PRSs) and disease end point PRSs (such as a CAD PRS) is that biomarker PRSs have a more direct mechanism and effect on clinical outcomes. The CAD PRS was based on a case-control setting of individuals with or without a CAD diagnosis with a risk 
Table 2. CAD Prediction With Lipid and CAD PRSs

\begin{tabular}{|c|c|c|c|c|c|}
\hline Predictors & AUC Unadjusted & AUC Baseline & AUC Full & OR $(95 \% \mathrm{Cl})$ Full & $P$ Value \\
\hline PRS $_{\text {LDLCC }}$ & 0.758 & 0.874 & 0.875 & $1.17(1.15-1.20)$ & $<2 \times 10^{-16}$ \\
\hline $\mathrm{PRS}_{\mathrm{TG}}$ & 0.757 & 0.874 & 0.875 & $1.12(1.09-1.14)$ & $<2 \times 10^{-16}$ \\
\hline $\mathrm{PRS}_{\mathrm{CAD}}$ & 0.767 & 0.874 & 0.879 & $1.43(1.40-1.46)$ & $<2 \times 10^{-16}$ \\
\hline $\mathrm{PRS}_{\mathrm{CAD}}+\mathrm{PRS}_{\text {LDLCC }}$ & 0.769 & 0.874 & 0.879 & & \\
\hline $\mathrm{PRS}_{\mathrm{CAD}}$ & & & & $1.41(1.38-1.44)$ & $<2 \times 10^{-16}$ \\
\hline PRS $_{\text {LDLCC }}$ & & & & $1.13(1.11-1.16)$ & $<2 \times 10^{-16}$ \\
\hline $\mathrm{PRS}_{\mathrm{CAD}}+\mathrm{PRS}_{\mathrm{TG}}$ & 0.768 & 0.874 & 0.879 & & \\
\hline $\mathrm{PRS}_{\mathrm{CAD}}$ & & & & $1.42(1.39-1.45)$ & $<2 \times 10^{-16}$ \\
\hline $\mathrm{PRS}_{\mathrm{TG}}$ & & & & $1.09(1.07-1.12)$ & $1.29 \times 10^{-15}$ \\
\hline $\mathrm{PRS}_{\mathrm{CAD}}+\mathrm{PRS}_{\mathrm{LDL}-\mathrm{C}}+\mathrm{PRS}_{\mathrm{TG}}$ & 0.769 & 0.874 & 0.879 & & \\
\hline $\mathrm{PRS}_{\mathrm{CAD}}$ & & & & $1.41(1.37-1.44)$ & $<2 \times 10^{-16}$ \\
\hline PRS $_{\text {LDL-C }}$ & & & & $1.11(1.09-1.14)$ & $<2 \times 10^{-16}$ \\
\hline $\mathrm{PRS}_{\mathrm{TG}}$ & & & & $1.06(1.03-1.08)$ & $2.49 \times 10^{-6}$ \\
\hline
\end{tabular}

ORs and AUCs for CAD with continuous LDL-C, TG, and CAD PRSs as predictors estimated using logistic regression. Unadjusted refers to a model with the PRSs as predictors, baseline to a model with age and sex as predictors, and full to a model with PRSs as predictors adjusted for age and sex. All models also include the technical covariates (first ten principal components and genotyping batch). AUC indicates area under the receiver operating characteristic curve; $C A D$, coronary artery disease; LDL-C, low-density lipoprotein cholesterol; OR, odds ratio; PRS, polygenic risk score; and TG, triglycerides.

of misclassifications and correlates little with known risk factors, complicating its interpretation and clinical implications. ${ }^{17}$ As the genome remains constant throughout life, unlike measured individual lipid values, PRSs are independent of age, medical conditions, medication usage, diet, fasting state, and other constantly changing confounding factors.

Our study has several limitations. First, as FINRISK participants fasted for a minimum of 4 hours before measuring lipid profiles, our association estimates may have been attenuated particularly between the TG PRS and TG levels. The association between the TG PRS and CAD risk, however, remains unaffected by this. Second, because the Friedewald formula is invalid for individuals

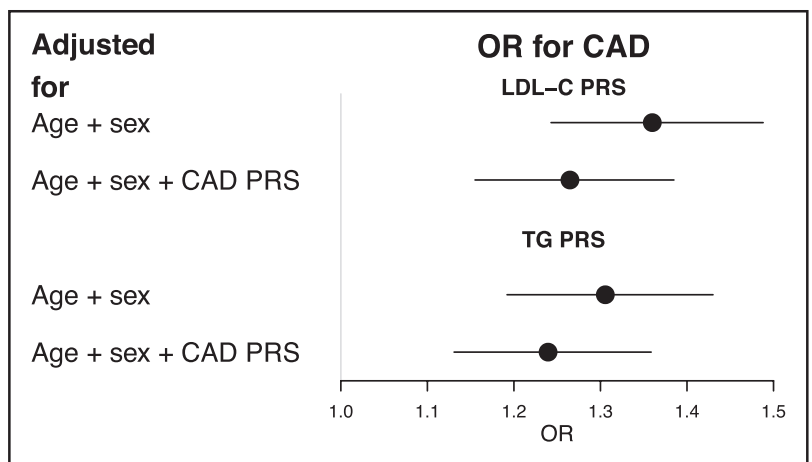

Figure 4. Odds ratios (ORs) for coronary artery disease (CAD) for those in the highest $5 \%$ of the polygenic risk scores (PRSs) compared with the remainder of the population with and without adjusting for the CAD PRS in the FinnGen project (FinnGen).

ORs were estimated using logistic regression. All models were additionally adjusted for age and sex. Horizontal lines represent 95\% Cls. LDL-C indicates low-density lipoprotein cholesterol; and TG, triglyceride. with TG $>4.52 \mathrm{mmol} / \mathrm{L}, 456$ (1.7\%) FINRISK samples were excluded from LDL-C analyses. ${ }^{18}$ Third, some variants included in the lipid PRSs are not specific to their primary lipids and have residual effects on others. Excepting a negative association between the TG PRSs and HDL (high-density lipoprotein) cholesterol, however, the PRSs had only minor associations with other than their primary lipids (Figure III in the Data Supplement). Fourth, our weights for the lipid PRSs came from the UK population and were tested in the Finnish population; our results may have limited accuracy in other ethnicities. Replication and validation in other cohorts with lipid measurements and populations is warranted in the future.

In summary, the CAD risk associated with a high polygenic load for LDL-C or TG -increasing genetic variants was proportional to their impact on lipid levels. In contrast with a PRS for CAD, the lipid PRSs point to a known and directly modifiable risk factor enabling more straightforward clinical translation. As PRSs can also be measured at any point in life, they provide powerful tools for prioritizing individuals for blood lipid panel screening and subsequent evidence-based intervention.

\section{ARTICLE INFORMATION}

Received August 14, 2019; accepted January 24, 2020.

\section{Affiliations}

Institute for Molecular Medicine Finland, Helsinki Institute of Life Science (HiLIFE) (P.R., J.T.R., N.J.M., Y.F., J.L., C.B., I.S., T.K., A.S.H., P.P., E.W., T.T., M.P., A.P., S.R.), Research Programs Unit, Diabetes and Obesity (S.S., M.-R.T.), Department of Public Health, Clinicum, Faculty of Medicine (M.P., S.R.), and Department of Mathematics and Statistics, Faculty of Science (M.P.), University of Helsinki, Helsinki, Finland. Department of Internal Medicine, Helsinki University Hospital, Helsinki, Finland (S.S.). Department of Internal Medicine, University of Michigan, Ann Arbor, MI (I.S.). Department of Public Health Solutions, Finnish Institute for Health and Welfare, Helsinki, Finland (A.S.H., V.S.). Center for Neurobehavioral Genetics, 
Semel Institute for Neuroscience and Human Behaviour, University of California Los Angeles, CA (N.B.F.). Program in Medical and Population Genetics (A.P.) and Stanley Center for Psychiatric Research (A.P.), Broad Institute of MIT and Harvard, Cambridge, MA. Psychiatric and Neurodevelopmental Genetics Unit, Department of Psychiatry (A.P.), Analytic and Translational Genetics Unit, Department of Medicine (A.P.), and Department of Neurology (A.P.), Massachusetts General Hospital, Boston, MA. Clinical Research Institute HUCH, Ltd, Helsinki, Finland (M.-R.T.); and Broad Institute of MIT and Harvard, Cambridge, MA (S.R.).

\section{Acknowledgments}

We would like to thank Sari Kivikko, Huei-Yi Shen, and Ulla Tuomainen for management assistance. The National FINRISK Study (FINRISK) data used for the research were obtained from THL Biobank. We thank the THL DNA Laboratory for its skillful work to produce the DNA samples used in the genotyping work, which were used in this study. Part of the genotyping was performed by the Institute for Molecular Medicine Finland Technology Centre, University of Helsinki. We thank all study participants for their generous participation in FINRISK, the GeneRISK Study (GeneRISK), the FinnGen project (FinnGen), and the UK biobank (UKBB). Drs P. Ripatti and Rämö acknowledge support from the Doctoral Programme in Population Health, University of Helsinki, and Dr Rämö acknowledges support from the MD-PhD Programme, University of Helsinki. Following biobanks are acknowledged for collecting the FinnGen project samples: Auria Biobank (https://www.auria.fi/biopankki/en), THL Biobank (https://thl.fi/fi/web/thlbiopankki), Helsinki Biobank (https://www.terveyskyla.fi/helsinginbiopankki/en), Northern Finland Biobank Borealis (https://www.ppshp.fi/Tutkimus-ja-opetus/ Biopankki), Finnish Clinical Biobank Tampere (https://www.tays.fi/en-US/Research_and_development/Finnish_Clinical_Biobank_Tampere), Biobank of Eastern Finland (https://ita-suomenbiopankki.fi/), Central Finland Biobank (https:// www.ksshp.fi/fi-Fl/Potilaalle/Biopankki), Finnish Red Cross Blood Service Biobank (https://www.bloodservice.fi/Research\%20Projects/biobanking). This research has been conducted using the UK Biobank Resource under Application Number 22627. The content is solely the responsibility of the authors and does not necessarily represent the official views of the National Institutes of Health.

\section{Sources of Funding}

This work was supported by the National Institutes of Health (grant No. HL113315 to Drs S. Ripatti, Taskinen, and Palotie); Finnish Foundation for Cardiovascular Research (to Drs S. Ripatti, Salomaa, Taskinen, and Palotie); Sigrid Jusélius Foundation (to Drs S. Ripatti and Taskinen); Biocentrum Helsinki (to Dr S. Ripatti); European Union (EU) Seventh Framework Program (grant No 305707 to Dr Taskinen); Helsinki University Central Hospital Research Funds (to Dr Taskinen); Leducq Foundation (to Dr Taskinen); Ida Montin Foundation (to Dr P. Ripatti); European Atherosclerosis Society (to Dr P. Ripatti); MD/PhD Program of the Faculty of Medicine, University of Helsinki (to Dr Rämö); Doctoral Programme in Population Health, University of Helsinki (to Drs Rämö and P. Ripatti) Finnish Medical Foundation (to Dr Rämö); Emil Aaltonen Foundation (to Drs P. Ripatti and Rämö); Biomedicum Helsinki Foundation (to Dr Rämö); Academy of Finland Center of Excellence in Complex Disease Genetics (grant No. 213506 , 129680, and 312062 to Dr S. Ripatti and grant No. 312076 to Dr Pirinen); Horizon 2020 Research and Innovation Programme (the ePerMed project [ePerMed] grant No. 692145 to Dr S. Ripatti); Helsinki Institute of Life Science (HiLIFE) Fellow grants 2017-2020 (to Dr S. Ripatti); and the Academy of Finland (grant No. 298149 to Dr Surakka, grant No. 288509 to Dr Pirinen, grant No. 321356 to Dr Havulinna, and grant No. 251217 and 285380 to Dr S. Ripatti). The FinnGen project is funded by 2 grants from Business Finland (HUS 4685/31/2016 and $\mathrm{UH}$ 4386/31/2016) and 9 industry partners (AbbVie, AstraZeneca, Biogen, Celgene, Genentech, GlaxoSmithKline, Merck Sharp \& Dohme Pfizer, and Sanofi). The funders had no role in study design, data collection and analysis, decision to publish, or preparation of the manuscript.

\section{Disclosures}

Dr Palotie is a member of the Pfizer Genetics Scientific Advisory Panel. Dr S. Ripatti holds a HiLIFE Fellowship. Dr Salomaa has participated in a conference trip sponsored by Novo Nordisk and received an honorarium from the same source for participating in an advisory board meeting. He also has ongoing research collaboration with Bayer, Ltd. The other authors report no conflicts.

\section{REFERENCES}

1. Di Angelantonio E, Sarwar N, Perry P, Kaptoge S, Ray KK, Thompson A Wood AM, Lewington S, Sattar N, Packard CJ, et al; Emerging Risk Factors Collaboration. Major lipids, apolipoproteins, and risk of vascular disease. JAMA. 2009;302:1993-2000. doi: 10.1001/jama.2009.1619
2. Fulcher J, O'Connell R, Voysey M, Emberson J, Blackwell L, Mihaylova B, Simes J, Collins R, Kirby A, Colhoun H, et al; Cholesterol Treatment Trialists' (CTT) Collaboration. Efficacy and safety of LDL-lowering therapy among men and women: meta-analysis of individual data from 174,000 participants in 27 randomised trials. Lancet. 2015;385:1397-1405. doi: 10.1016/S0140-6736(14)61368-4

3. Do R, Willer CJ, Schmidt EM, Sengupta S, Gao C, Peloso GM, Gustafsson S, Kanoni S, Ganna A, Chen J, et al. Common variants associated with plasma triglycerides and risk for coronary artery disease. Nat Genet. 2013;45:13451352. doi: $10.1038 / n g .2795$

4. Blood I, Crosby J, Peloso GM, Auer PL, Crosslin DR, Stitziel NO, Lange LA, $\mathrm{Lu} Y$, et al; Tg, Hdl Working Group of the Exome Sequencing Project NHL. Loss-of-function mutations in APOC3, triglycerides, and coronary disease. N Engl J Med. 2014;371:22-31. doi: 10.1056/NEJMoa1307095

5. Nordestgaard BG, Varbo A. Triglycerides and cardiovascular disease. Lancet. 2014;384:626-635. doi: 10.1016/S0140-6736(14)61177-6

6. Hegele RA, Ginsberg HN, Chapman MJ, Nordestgaard BG, Kuivenhoven JA, Averna M, Borén J, Bruckert E, Catapano AL, Descamps OS, et al; European Atherosclerosis Society Consensus Panel. The polygenic nature of hypertriglyceridaemia: implications for definition, diagnosis, and management. Lancet Diabetes Endocrinol. 2014;2:655-666. doi: 10.1016/S2213-8587(13)70191-8

7. Bhatnagar D, Soran H, Durrington PN. Hypercholesterolaemia and its management. BMJ. 2008;337:a993. doi: 10.1136/bmj.a993

8. Talmud PJ, Shah S, Whittall R, Futema M, Howard P, Cooper JA, Harrison SC, Li K, Drenos F, Karpe F, et al. Use of low-density lipoprotein cholesterol gene score to distinguish patients with polygenic and monogenic familial hypercholesterolaemia: a case-control study. Lancet. 2013;381:1293-1301. doi: 10.1016/S0140-6736(12)62127-8

9. Ripatti P, Rämö JT, Söderlund S, Surakka I, Matikainen N, Pirinen M, Pajukanta P, Sarin AP, Service SK, Laurila PP, et al. The contribution of GWAS loci in familial dyslipidemias. PLoS Genet. 2016;12:e1006078. doi: 10.1371/journal.pgen.1006078

10. Khera AV, Won HH, Peloso GM, Lawson KS, Bartz TM, Deng X, van Leeuwen EM, Natarajan P, Emdin CA, Bick AG, et al. Diagnostic yield and clinical utility of sequencing familial hypercholesterolemia genes in patients with severe hypercholesterolemia. J Am Coll Cardiol. 2016;67:2578-2589. doi: 10.1016/j.jacc.2016.03.520

11. Kathiresan S, Melander O, Anevski D, Guiducci C, Burtt NP, Roos C, Hirschhorn JN, Berglund G, Hedblad B, Groop L, et al. Polymorphisms associated with cholesterol and risk of cardiovascular events. $N$ Engl J Med. 2008;358:1240-1249. doi: 10.1056/NEJMoa0706728

12. Bhatt DL, Steg PG, Miller M, Brinton EA, Jacobson TA, Ketchum SB, Doyle RT Jr, Juliano RA, Jiao L, Granowitz C, et al; REDUCE-IT Investigators. Cardiovascular risk reduction with icosapent ethyl for hypertriglyceridemia. N Engl J Med. 2019;380:11-22. doi: 10.1056/ NEJMoa1812792

13. Catapano AL, Graham I, De Backer G, Wiklund O, Chapman MJ, Drexel H, Hoes AW, Jennings CS, Landmesser U, Pedersen TR, et al; ESC Scientific Document Group. 2016 ESC/EAS guidelines for the management of dyslipidaemias. Eur Heart J. 2016;37:2999-3058. doi: 10.1093/eurheartj/ehw272

14. Borodulin K, Tolonen H, Jousilahti P, Jula A, Juolevi A, Koskinen S, Kuulasmaa K, Laatikainen T, Männistö S, Peltonen M, et al. Cohort profile: the National FINRISK Study. Int J Epidemiol. 2018;47:696-696i. doi: 10.1093/ije/dyx239

15. Abul-Husn NS, Manickam K, Jones LK, Wright EA, Hartzel DN, GonzagaJauregui C, O'Dushlaine C, Leader JB, Lester Kirchner H, Lindbuchler DM, et al. Genetic identification of familial hypercholesterolemia within a single U.S. health care system. Science. 2016;354:aaf7000. doi: 10.1126/science.aaf7000

16. Lahtinen AM, Havulinna AS, Jula A, Salomaa V, Kontula K. Prevalence and clinical correlates of familial hypercholesterolemia founder mutations in the general population. Atherosclerosis. 2015;238:64-69. doi: 10.1016/j.atherosclerosis.2014.11.015

17. Khera AV, Chaffin M, Aragam KG, Haas ME, Roselli C, Choi SH, Natarajan P, Lander ES, Lubitz SA, Ellinor PT, et al. Genome-wide polygenic scores for common diseases identify individuals with risk equivalent to monogenic mutations. Nat Genet. 2018;50:1219-1224. doi: 10.1038/s41588-018-0183-z

18. Friedewald WT, Levy RI, Fredrickson DS. Estimation of the concentration of low-density lipoprotein cholesterol in plasma, without use of the preparative ultracentrifuge. Clin Chem. 1972;18:499-502. 